\title{
EVALUACIÓN DEL LIDERAZGO PEDAGÓGICO DEL DIRECTOR Y SU \\ RELACIÓN CON EL DESEMPEÑO DOCENTE EN LA INSTITUCIÓN \\ EDUCATIVA N'2069 SANTA ROSA. UGEL 04. \\ DISTRITO DE PUENTE PIEDRA. REGIÓN LIMA 2017.
}

\begin{abstract}
EVALUATION OF PEDAGOGICAL LEADERSHIP OF THE DIRECTOR AND HIS
RELATIONSHIP WITH TEACHING PERFORMANCE IN THE INSTITUCIÓN

EDUCATIVA N²069 SANTA ROSA. UGEL 04 PUENTE PIEDRA DISTRICT.
\end{abstract}

LIMA REGION 2017.

AVALIAÇÃO DA LIDERANÇA PEDAGÓGICA DO DIRETOR E SUA RELAÇÃO COM O
DESEMPENHO ENSINO INSTITUIÇÃO EDUCATIVA N 2069 SANTA ROSA. UGEL 04
PUENTE PIEDRA DISTRITO. REGIÃO DE LIMA 2017.

ISSN: 2617-619X

Resumen.- La investigación científica que hemos realizado es básica, correlacional causal no experimental, los factores de estudio son dos: Evaluación del Liderazgo Pedagógico del Director y su Relación con el Desempeño Docente en la Institución Educativa N 2069 Santa Rosa. UGEL 04. Distrito de Puente Piedra. Región Lima 2017. La población está conformada por 2 Directivos y 56 Docentes.

El diseño de la investigación es transaccional, correlacional, causal. bivariada, transversal. Para la recolección de datos se confeccionaron dos cuestionarios: uno para

\footnotetext{
${ }^{1}$ Maestrista de Educación con mención en Evaluación y Acreditación de la Calidad de la Educación de la Universidad Nacional Mayor de San Marcos.

${ }^{2}$ Dr. en Educación. Catedrática Principal de la facultad de Educación de la Universidad Nacional Mayor de San Marcos. Lima-Perú.
} 
OBREGÓN CASTILEJO, Liz Castillejo; Carlos BARRIGA HERNÁNDEZ - Evaluación del liderazgo pedagógico del director y su relación con el desempeño docente en la Institución Educativa N²069 Santa Rosa. UGELI 04. Distrito de Puente Piedra. Región lima 2017. Rev. Igobernanza. Setiembre 2018. Vol.1/N³, pp. 28-38.

la variable Liderazgo pedagógico del director y el otro para a variable evaluación del desempeño docente, que se aplicó a los 2 Directivos y 56 Docentes de la muestra.

Los instrumentos cumplen con las cualidades de validez y confiabilidad. El estudio plantea la siguiente hipótesis de investigación: Evaluación y Liderazgo Pedagógico del Director y su Relación con el Desempeño Docente en la Institución Educativa N²069 Santa Rosa. UGEL 04. Distrito de Puente Piedra. Región Lima 2017.

Los resultados obtenidos nos permiten rechazar la hipótesis nula y aceptar la hipótesis investigadas pues se ha encontrado en una prueba no paramétrica por lo tanto se aplicó Prueba de Kruskal-Wallis para observar la relación significativa entre el liderazgo pedagógico y el desempeño docente con un nivel de significancia del $95 \%$ obteniendo un p_valor de 0.00 , además se aplicó la prueba de regresión lineal y se obtuvo que el liderazgo pedagógico se relaciona en un $42,4 \%$ con el desempeño docente por lo tanto se rechaza la hipótesis nula y se acepta la hipótesis alterna de investigación.

Palabras Clave: Evaluación Liderazgo Pedagógico del Director - Desempeño Docente - Puente Piedra - 2017

Abtract.- The scientific research we have conducted is basic, non-experimental causal correlation, the study factors are two: Evaluation of the Pedagogical Leadership of the Director and his Relation with the Teaching Performance in the Educational Institution N $\odot 2069$ Santa Rosa. UGEL 04. Puente Piedra District. Lima Region 2017. The population is made up of 2 Directors and 56 Teachers.

The research design is transactional, correlational, causal. bivariate, transversal. Two questionnaires were drawn up for the data collection: one for the variable Pedagogical Leadership of the director and the other for a variable evaluation of the teaching performance, which was applied to the 2 Directors and 56 Teachers of the sample.

The instruments comply with the qualities of validity and reliability. The study proposes the following research hypothesis: Evaluation and Pedagogical Leadership of 
the Director and his Relation with the Teaching Performance in the Educational Institution N ${ }^{\circ} 2069$ Santa Rosa. UGEL 04. Puente Piedra District. Lima Region 2017.

The results obtained allow us to reject the null hypothesis and accept the hypotheses investigated because it has been found in a non-parametric test therefore Kruskal-Wallis test was applied to observe the significant relationship between pedagogical leadership and teaching performance with a level of significance of $95 \%$ obtaining a value of 0.00 , in addition the lineal regression test was applied and it was obtained that the pedagogical leadership is related in a $42.4 \%$ with the teaching performance therefore the null hypothesis is rejected and the hypothesis is accepted alternate research

Keywords: Evaluation Pedagogical Leadership Evaluation - Teaching Performance Puente Piedra - 2017

Introducción.- La educación nacional se encuentra en una situación crítica. Los estándares de medición y evaluación de la calidad educativa tanto en el mundo como en el Perú nos indican que los niños y adolescentes no logran las competencias básicas en las áreas de comunicación integral y desarrollo del pensamiento lógico.

Los resultados del proceso de aprendizaje obligan a los responsables de la educación y a todos los que están comprometidos con el desarrollo educativo, a identificar en la relación director-docente-estudiante, cuál es el factor determinante que está influyendo para que este hecho educativo sea parte de nuestra realidad, y una vez identificado, impulsar y orientar la toma de decisiones para corregir las deficiencias en logros de aprendizaje.

En los resultados escolares, queda absolutamente claro el papel central que cumple tanto el docente, como el responsable de la gestión, vale decir el director, a través de sus actitudes y capacidades profesionales. Si bien existen factores asociados que influyen en los resultados descritos, es el factor escuela en quien recae la mayor responsabilidad, por ello constituye el foco de atención de cualquier política de mejora. 
Esta investigación que se realizó en las Institución Educativa $N^{\circ} 2069$ Santa Rosa. UGEL 04. Distrito de Puente Piedra. Región Lima 2017, buscará encontrar la relación entre el liderazgo pedagógico del director y la evaluación del desempeño de los docentes, traducidos en resultados de aprendizajes en los estudiantes.

Dado que en la actualidad, explica Castillo (2008), las instituciones escolares se conciben como una empresa, su modelo de gestión debe dirigir sus acciones hacia el logro de los objetivos propuestos, pues se considera a sus directivos como gerentes, quienes deben reunir un conjunto de requisitos y cualidades, entre las que cabe mencionar el desarrollo de un liderazgo que le permita no sólo cumplir con el ejercicio de las funciones gerenciales básicas omo planificación, organización, dirección, control, sino también conducir al talento humano de su organización educativa para la optimización de su desempeño laboral como elemento esencial para lograr las metas propuestas.

Medina (2010), considera que resulta prioritario de quienes ocupan cargos directivos el conocimiento necesario en materia gerencial, el cual le proporcione las herramientas necesarias para conducir adecuadamente la organización de manera de propiciar un mejoramiento del desempeño laboral del docente.

De acuerdo con lo antes expuesto, el director debe estar capacitado para tomar las decisiones más pertinentes, ser apto y capaz de motivar, accionar, gerenciar, asignar responsabilidades e inspirar respeto, confianza, cooperación, elementos claves para influir en el comportamiento de los docentes mejorando su desempeño laboral. Al respecto, Laval (2004) señala "en el campo educativo se han ido insertando estos conceptos buscando un punto de encuentro entre funciones gerenciales y procesos educativos, esta cohesión ha permitido que los autores que se han dedicado al estudio de procesos gerenciales en el campo educativo" (p.23).

Es por esa razón, que este subsistema requiere de directivos con conocimientos gerenciales que le permitan el logro de acciones para conducir con acierto las instituciones guiando al personal a mejorar su desempeño, así como poseedor de una actitud positiva ante los cambios educativos y con disposición a una formación 
permanente. Esto se sustenta en lo expresado por Sambrano y Steiner (2007) "la educación exige profesionales capaces de continuar su actualización. Aprendices permanentes con habilidades para aprender-aprender, capacidad de producir o utilizar conocimientos" (p.19).

Los señalamientos anteriores permiten inferir que la gestión gerencial del directivo es una pieza clave para una excelente práctica educativa, al configurar un proceso educativo el cual cumpla con las exigencias sociales de las organizaciones, pues según Alles (2006):

Las investigaciones y teorías del liderazgo que vienen desde el ámbito empresarial y han sido llevadas al contexto escolar se enfocan en los rasgos o las conductas y/o acciones de los líderes, así como en las conductas en relación con las situaciones en donde se ejerce el liderazgo, los seguidores y los subordinados (Agüera, 2006, en López-Gorosave, Slater, \& García-Garduño, 2010). Algunas teorías han tipificado los estilos de liderazgo; por ejemplo, la teoría conductual refiere los estilos autocrático, democrático y permisivo; la situacional habla del liderazgo empobrecido, de tarea, punto medio, club de campo y de equipo. Posteriormente, la teoría transformacional caracteriza el liderazgo transformacional y transaccional (LópezGorosave, Slater, \& García-Garduño, 2010, p. 36).

El liderazgo pedagógico, a nivel de escuelas, desempeña un rol altamente significativo en el desarrollo de cambios en las prácticas docentes, en la calidad de estas, y en el impacto que presentan sobre la calidad del aprendizaje de los alumnos (Anderson, 2010). En el liderazgo de las escuelas eficaces y que mejoran... los líderes se centran más en aspectos relacionados con la enseñanza y el aprendizaje, que en otros (de tipo administrativo) (Muijs, 2003); el foco está centrado principalmente en la gestión de los aprendizajes y la mejora de las prácticas docentes.

\section{Objetivo General}

- Determinar en qué medida se relaciona la Evaluación del Liderazgo Pedagógico del Director y su Relación con el Desempeño Docente en la Institución 
Educativa N²069 Santa Rosa. UGEL 04. Distrito de Puente Piedra. Región Lima 2017.

\section{Objetivos Específicos}

1. Identificar en qué medida se relaciona la evaluación del liderazgo pedagógico del director en la planificación del trabajo docente en las Institución Educativa N²069 Santa Rosa. UGEL 04. Distrito de Puente Piedra. Región Lima 2017.

2. Determinar si la evaluación del liderazgo pedagógico del director se relaciona en los procesos de enseñanza docente en las Institución Educativa № 2069 Santa Rosa. UGEL 04. Distrito de Puente Piedra. Región Lima 2017.

3. Establecer si la evaluación del liderazgo pedagógico del director se relaciona con la profesionalidad y la identidad docente en las Institución Educativa $\mathrm{N}^{\circ} 2069$ Santa Rosa. UGEL 04. Distrito de Puente Piedra. Región Lima 2017.

\section{Material y Método:}

El estudio de la presente investigación tiene dos variables; la variable $\mathrm{Y}$ (Evaluación Liderazgo pedagógico del director) es el efecto y la causa es la variable X (Desempeño docente).

Por el nivel de conocimientos, es explicativa porque da respuestas al por qué de la investigación y establece relaciones entre las variables para conocer la estructura y factores que intervienen en el proceso.

El diseño aplicable es correlacional explicativa, el cual permitirá la relación entre las variables del estudio, se representa de la siguiente manera:

$\mathrm{r}$

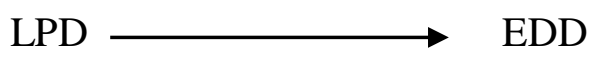

$$
\begin{array}{lll}
\operatorname{LPD}(\mathrm{X}) \quad= & \text { Evaluación Liderazgo pedagógico del director } \\
\operatorname{EDD}(\mathrm{Y}) \quad= & \text { Desempeño docente } \\
\mathrm{r} \quad=\text { Correlación } &
\end{array}
$$


OBREGÓN CASTILEJO, Liz Castillejo; Carlos BARRIGA HERNÁNDEZ - Evaluación del liderazgo pedagógico del director y su relación con el desempeño docente en la Institución Educativa N²069 Santa Rosa. UGELI 04. Distrito de Puente Piedra. Región lima 2017. Rev. Igobernanza. Setiembre 2018. Vol.1/N³, pp. 28-38. ISSN: 2617-619X

\section{Resultados y Discusión:}

Gráfico 1 . Tabla del desempeño docente y las diferencias de medias

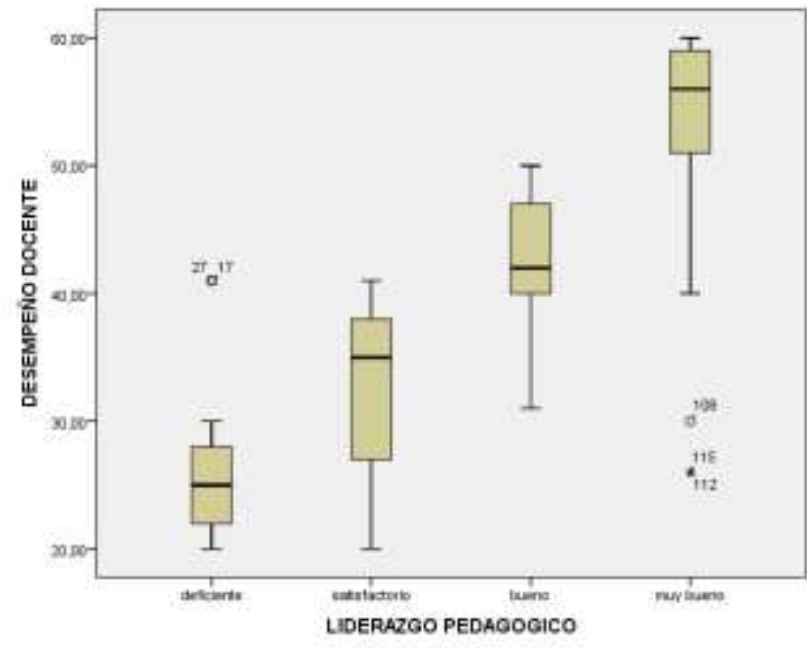

Fuente: Elaboración propia

En Gráfico 1, se observa que el rango promedio del desempeño docente que presenta un deficiente liderazgo pedagógico es 27,08; el rango promedio de los que presentan un liderazgo satisfactorio es 49,24.El rango promedio de los que presentan un liderazgo bueno en el desempeño docente es 80,82. El 108,86 posee un liderazgo muy bueno. Estos resultados nos indican que mientras mejor sea el liderazgo pedagógico mayor será la media.

Gráfico 2. Distribución porcentual de Competencias logradas en el desempeño docente

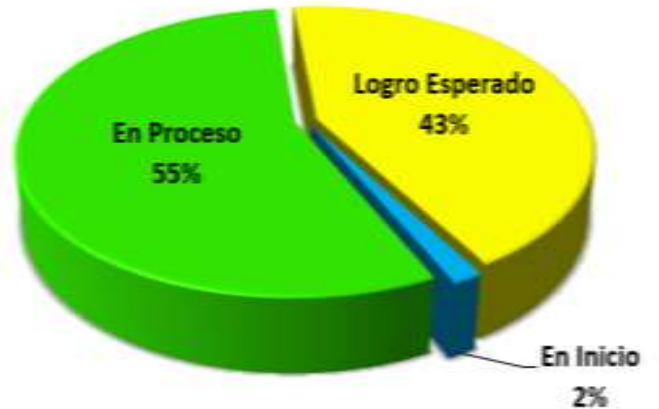

Fuente: Elaboración propia 
OBREGÓN CASTILEJO, Liz Castillejo; Carlos BARRIGA HERNÁNDEZ - Evaluación del liderazgo pedagógico del director y su relación con el desempeño docente en la Institución Educativa N²069 Santa Rosa. UGELI 04. Distrito de Puente Piedra. Región lima 2017. Rev. Igobernanza. Setiembre 2018. Vol.1/N³, pp. 28-38.

En Gráfico 2, se observa que el grupo mayoritario de docentes presentan un desempeño de competencias logradas en el nivel proceso con un 55,3\%, 31 docentes presentan un desempeño de logro esperado con 43,2 y con un 55,3\%. En el nivel inicio, se encuentra al $1,5 \%$ de docentes. Estos resultados nos indican que la mayoría de docentes alcanzan un nivel de logro esperado.

Gráfico 3 . Distribución porcentual del desempeño docente del liderazgo deficiente

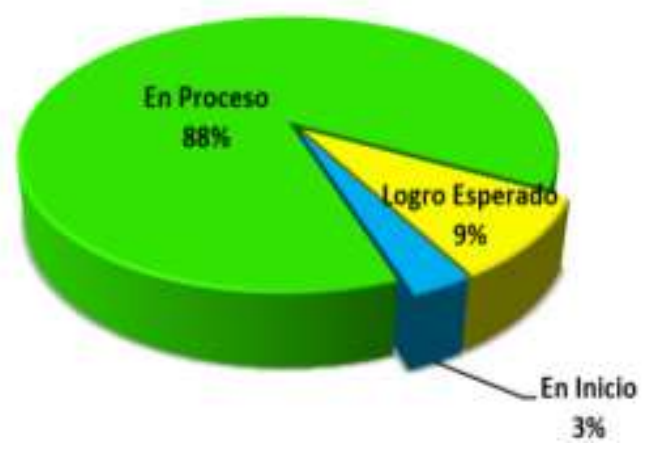

Fuente: Elaboración propia

En Gráfico 3, se observa que el grupo mayoritario de docentes que presentan un liderazgo pedagógico deficiente presentan un desempeño en el nivel proceso con un $87,9 \%$, los docentes que presentan un desempeño de logro esperado representan al 9,1\% y con un $3 \%$ se encuentran los docentes con un nivel de liderazgo deficiente en el nivel inicio. Estos resultados nos indican que la mayoría de docentes alcanzan un nivel en proceso.

Gráfico 4 Desempeño Docente Del Liderazgo Satisfactorio

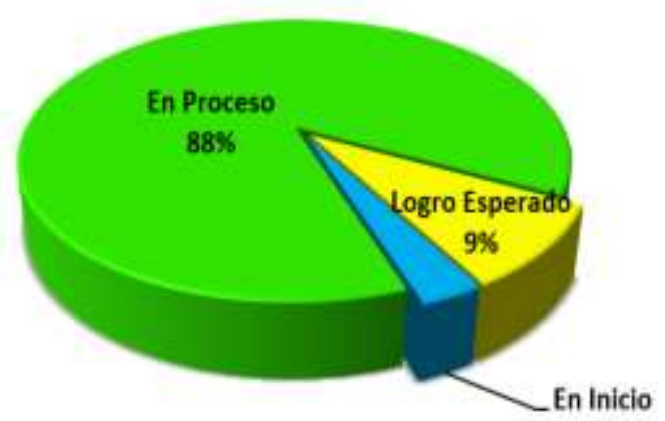

$3 \%$

Fuente: Elaboración propia 
OBREGÓN CASTILEJO, Liz Castillejo; Carlos BARRIGA HERNÁNDEZ - Evaluación del liderazgo pedagógico del director y su relación con el desempeño docente en la Institución Educativa N²069 Santa Rosa. UGELI 04. Distrito de Puente Piedra. Región lima 2017. Rev. Igobernanza. Setiembre 2018. Vol.1/N³, pp. 28-38.

En Gráfico 4, se observa que el grupo mayoritario de docentes que presentan un liderazgo pedagógico satisfactorio presentan un desempeño en el nivel proceso con un $87,9 \%$, los docentes que presentan un desempeño de logro esperado representan al 9,1\% y con un $3 \%$ se encuentran los docentes con un nivel de liderazgo satisfactorio en el nivel inicio. Estos resultados nos indican que la mayoría de docentes alcanzan un nivel en proceso.

\section{Gráfico 5 Desempeño Docente Del Liderazgo Bueno}

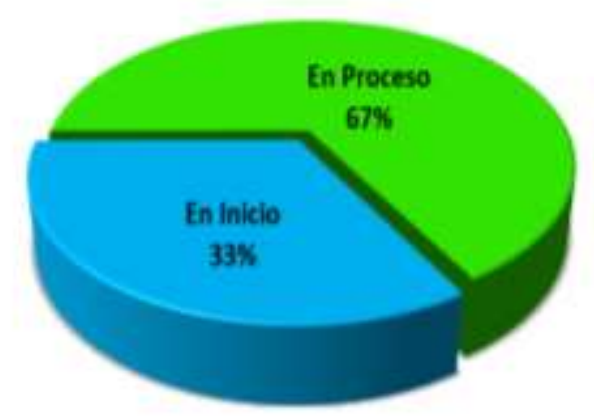

Fuente: Elaboración propia

En Gráfico 5, se observa que el grupo mayoritario de docentes que presentan un liderazgo pedagógico bueno presentan un desempeño en el nivel logro esperado con un $66,7 \%$, los docentes que presentan un desempeño en proceso representan al 33,3\%. Estos resultados nos indican que la mayoría de docentes alcanzan un nivel de logro esperado.

Gráfico 6. Desempeño Docente Del Liderazgo Muy Bueno

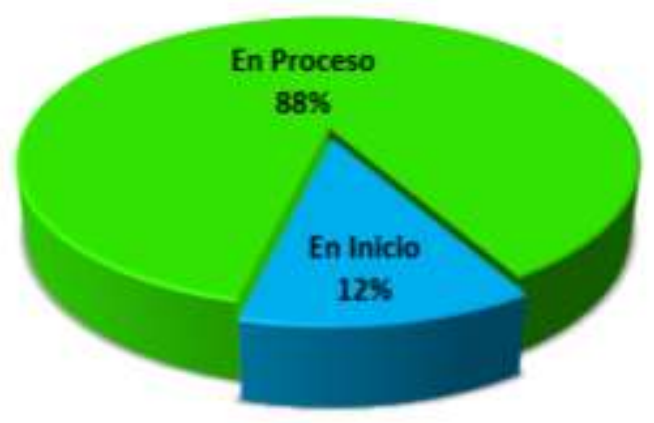

Fuente: Elaboración propia 
En Gráfico 6, se observa que el grupo mayoritario de docentes que presentan un liderazgo pedagógico muy bueno presentan un desempeño en el nivel logro esperado con un $87,9 \%$, los docentes que presentan un desempeño de logro esperado representan al $12,1 \%$. Estos resultados nos indican que la mayoría de docentes alcanzan un nivel de logro esperado.

\section{Conclusiones}

Primero.- Se ha demostrado que el liderazgo pedagógico del director influye en la evaluación del desempeño docente en las instituciones educativas del nivel secundario de la Institución Educativa $\mathrm{N}^{\circ} 2069$ Santa Rosa - UGEL 04, según la Prueba de Kruskal-Wallises de 0,00 asociación de las variables y siendo altamente significativo. Además, según la regresión lineal el liderazgo influye en 42,4\% y se acepta la hipótesis: El liderazgo pedagógico del director influye en la evaluación del desempeño docente en las instituciones educativas del nivel secundario de la Institución Educativa $N^{\circ} 2069$ Santa Rosa - UGEL 04

Segundo.- Se ha demostrado que el liderazgo pedagógico del director influye en la planificación del trabajo docente en las Institución Educativa $N^{\circ} 2069$ Santa Rosa. UGEL 04. Distrito de Puente Piedra. Región Lima 2017, según la Prueba de KruskalWallises de 0,00 asociación de las variables y siendo altamente significativo. Además, según la regresión lineal el liderazgo influye en 37,1\% y se acepta la hipótesis: El liderazgo pedagógico del director influye en la planificación del trabajo docente en las Institución Educativa N 2069 Santa Rosa. UGEL 04. Distrito de Puente Piedra. Región Lima 2017.

Tercero.- Se ha demostrado que el liderazgo pedagógico del director influye en los procesos de enseñanza docente en las Institución Educativa N²069 Santa Rosa. UGEL 04. Distrito de Puente Piedra. Región Lima 2017., según la Prueba de Kruskal-Wallises de 0,00 asociación de las variables y siendo altamente significativo. Además, según la regresión lineal el liderazgo influye en 34,6\% y se acepta la hipótesis: El liderazgo pedagógico del director influye en la conducción de los procesos de enseñanza en las 
OBREGÓN CASTILEJO, Liz Castillejo; Carlos BARRIGA HERNÁNDEZ - Evaluación del liderazgo pedagógico del director y su relación con el desempeño docente en la Institución Educativa N²069 Santa Rosa. UGELI 04. Distrito de Puente Piedra. Región lima 2017. Rev. Igobernanza. Setiembre 2018. Vol.1/N³, pp. 28-38. ISSN: 2617-619X

Institución Educativa N 2069 Santa Rosa. UGEL 04. Distrito de Puente Piedra. Región Lima 2017.

Cuarto.- Se ha demostrado que el liderazgo pedagógico del director influye en la profesionalidad y la identidad docente en las Institución Educativa $N^{\circ} 2069$ Santa Rosa. UGEL 04. Distrito de Puente Piedra. Región Lima 2017, según la Prueba de KruskalWallises de 0,00 asociación de las variables y siendo altamente significativo. Además, según la regresión lineal el liderazgo influye en 29,9\% y se acepta la hipótesis: El liderazgo pedagógico del director influye en el desarrollo de la profesionalidad y la identidad docente en las Institución Educativa N² 2069 Santa Rosa. UGEL 04. Distrito de Puente Piedra. Región Lima 2017.

\section{Referencias Bibliográficas.}

Alles, M. (2006) Desarrollo del talento humano basado en competencias. Buenos Aires: Granica

Chiavenato, I. (2009) Administración en los Nuevos Tiempos. México: Mc Grw Hill Laval (2004) Gestión y Calidad Educativa. Madrid: Narcea

López, Z (2003). Ética de la profesión académica en la época global, en Hirsch, A. \& López Zavala, R. ética profesional e identidad institucional. UAS: México, (p.

MINEDU. (2012). Marco del Buen desempeño docente, aportes y comentarios. Documento de trabajo, Lima MINEDU, (p. 13)

Medina, V. (2010) Competencias del asesor técnico de Educación Física desde la perspectiva del liderazgo efectivo. Trabajo de Grado no publicado. Universidad del Zulia. Maracaibo

Muijs, D. (2003). La mejora y la eficacia de las escuelas en zonas desfavorecidas: resumen de resultados de investigación. Revista Electrónica Iberoamericana sobre calidad, Eficacia y Cambio en Educación, 1 (2), (p. 63)

Sambrano y Steiner (2007) Estrategias educativas para docentes y padres del Siglo XXI. Caracas: Alfa 